\title{
RUIN PROBABILITY UNDER COMPOUND POISSON MODELS WITH RANDOM DISCOUNT FACTOR
}

\author{
KaI W. Ng and HaILIANg Yang \\ Department of Statistics and Actuarial Science \\ The University of Hong Kong \\ Pokfulam Road, Hong Kong \\ E-mail: hlyang@hkusua.hku.hk \\ LIHONG ZHANG \\ Department of Finance \\ Tsinghua University \\ Beijing, People's Republic of China
}

\begin{abstract}
In this article, we consider a compound Poisson insurance risk model with a random discount factor. This model is also known as the compound filtered Poisson model. By using some stochastic analysis techniques, a convergence result for the discounted surplus process, an expression for the ruin probability, and the upper bounds for the ruin probability are obtained.
\end{abstract}

\section{INTRODUCTION}

Recently, the interplay between actuarial science and finance has attracted much attention. One important question is, "What happens if we consider the investment risk in the insurance risk model?" There are many recent articles in actuarial science literature which tackle this problem. Sundt and Teugels [13] considered a compound Poisson model with a constant interest rate. Renewal-type equations satisfied by the ruin probability, asymptotic expression, and the upper bounds for the ruin probability were obtained. Yang and Zhang [15] extended the work in Sundt and Teugels [13] and considered the joint distribution of the surplus immediately before and after ruin. Yang [14] considered a discrete model with a constant interest rate. By using 
martingale inequalities, both exponential and nonexponential bounds were obtained. Paulsen [10] and Norberg [9] used diffusion models with stochastic interest incomes. Paulsen and Gjessing [11] provided some results for a model with stochastic investment incomes. Asmussen [2] summarized some recent works on ruin probability with investment income.

Delbaen and Haezendonck [5] considered the risk theory problems in an economic environment by discounting the value of the surplus from the current (or future) time to the initial time. The present article is in the spirit of Delbaen and Haezendonck [5], but whereas they used a deterministic discount factor, we will use a random discount factor. This is somewhat the same as when we consider the compound Poisson model with a stochastic interest rate. We call the model a filtered compound Poisson model.

This article is organized as follows. Section 2 provides the model and assumptions, Section 3 obtains a convergence result for the discounted surplus process, Section 4 gives an expression for the ruin probability, Section 5 discusses the constant interest force and exponential claim case, Section 6 obtains some upper bounds for the ruin probability, and the last section deals with the case of negative security loading.

\section{THE MODEL}

The classical risk model can be described as follows:

$$
U(t)=u+c t-\sum_{i=1}^{N(t)} X_{i}
$$

where $U(t)$ denotes the surplus at time $t, u$ denotes the initial surplus, $c$ denotes the premium rate in a unit time, $X_{i}$ denotes the amount of the $i$ th claim, and $N(t)$ denotes the number of claims occurring in the time interval $(0, t]$. It is assumed that $\{N(t)$; $t>0\}$ is a homogeneous Poisson process with an intensity of $\lambda$. It is also assumed that the claim amounts are independent of the claim number process and are positive and mutually independent and identically distributed (i.i.d.) with common distribution function $F$, where $F$ satisfies $F(0)=0$. Moreover, we assume that $F$ has a mean $p_{1}$ and

$$
p_{2}=\int_{0}^{+\infty} x^{2} d F(x)<+\infty
$$

We assume that

$$
c t=(1+\theta) \lambda p_{1} t=(1+\theta) E\left(\sum_{i=1}^{N(t)} X_{i}\right),
$$

where $\theta$ is called security loading. We assume that $\theta>0$ in the first part (up to Section 6) of this article, and we discuss the case where $\theta \leq 0$ in Section 7. 
In the literature, there are many articles dealing with the ruin problems for the classical risk model. However, in the real world, investment income plays a very important role. In this article, we consider the insurance risk model where the interest rate is random. Let $T_{i}$ denote the $i$ th claim time and let $W_{t}$ denote the discount factor. The discounted value of total claim amounts occurring in the time interval $(0, t]$ can then be described as

$$
S(t)=\sum_{i=1}^{N(t)} X_{i} W_{T_{i}}
$$

$S=\left\{S_{t}\right\}$ is called a filtered Poisson process. Using the properties of the filtered Poisson process (see Deng and Liang [6] or Snyder [12]), we have

$$
\begin{aligned}
E\left(\sum_{i=1}^{N(t)} X_{i} W_{T_{i}} \mid \sigma\left(W_{s}: 0 \leq s<t\right)\right) & =\lambda p_{1} \int_{0}^{t} W_{s} d s \\
\operatorname{Var}\left(\sum_{i=1}^{N(t)} X_{i} W_{T_{i}} \mid \sigma\left(W_{s}: 0 \leq s<t\right)\right) & =\lambda E\left(X_{i}^{2}\right) \int_{0}^{t} W_{s}^{2} d s,
\end{aligned}
$$

where $\sigma\left(W_{s}: 0 \leq s<t\right)$ denotes the $\sigma$-field generated by $\left\{W_{s}: 0 \leq s<t\right\}$.

Assuming that the interest rate is random (equivalently, the discount factor $W_{t}$ is random), similar to (2.1), the surplus process of an insurance company can then be described by the following model:

$$
U_{t}^{(W)}=u+(1+\theta) \lambda p_{1} \int_{0}^{t} W_{s} d s-\sum_{i=1}^{N(t)} X_{i} W_{T_{i}}
$$

where $U_{t}^{(W)}$ denotes the discounted value of the surplus (discounted from time $t$ to time 0 ) and all the other notations remain the same as earlier. We call model (2.5) the compound filtered Poisson model.

In this article, we make the following assumptions:

1. $\left\{W_{t}\right\},\left\{X_{i}\right\}$, and $\{N(t)\}$ are mutually independent.

2. $W_{0}=1$, and $W_{t}>0$ for all $t>0$, a.e.

The first assumption is a common one in actuarial science. Although, recently, there have been some works on the dependent risk in the actuarial literature, we do not consider this issue here. The second assumption states that the interest rate cannot be positive infinity. Our model here is very general. In practice, we usually assume that the interest rate is nonnegative at any time. This corresponds to $W_{t}>0$ and $W_{t}$ is nonincreasing for all $t>0$.

\section{CONVERGENCE OF $U_{t}^{(w)}$}

In this section, we will prove a convergence result. This convergence result will then be used in later sections to prove some useful results in the insurance risk theory. In this section, we assume that the discount factor $W_{t}$ satisfies the following assumption: 


$$
E\left(\int_{0}^{+\infty} W_{s} d s\right)=\frac{1}{\gamma_{1}}<+\infty .
$$

Under the above assumption, we have the following result.

THEOREM 1: Under assumption (3.1), there exists an integrable random variable $U_{\infty}^{(W)}$ such that

$$
\lim _{t \rightarrow+\infty} U_{t}^{(W)} \stackrel{\text { a.e. }}{=} U_{\infty}^{(W)}
$$

and

$$
E\left(U_{\infty}^{(W)}\right)=\frac{\theta \lambda p_{1}}{\gamma_{1}}+u,
$$

where a.e. means almost everywhere with respect to probability measure P. Moreover, the characteristic function of $U_{\infty}^{W}$ is given by

$$
\begin{aligned}
E\left(e^{i x U_{\infty}^{W}}\right)=E[ & \exp \left\{i x\left(u+\lambda p_{1} \theta \int_{0}^{+\infty} W_{s} d s\right)-\frac{1}{2} x^{2} \lambda p_{2} \int_{0}^{+\infty} W_{s}^{2} d s\right. \\
& \left.\left.+\lambda \int_{0}^{+\infty} \int_{0}^{x W_{t}}\left(x W_{t}-s\right) E\left[X^{2}\left(1-e^{-i s X}\right)\right] d s d t\right\}\right] .
\end{aligned}
$$

Proof: Let

$$
M_{t}=\lambda p_{1} t-\sum_{i=1}^{N(t)} X_{i} .
$$

Then, $M_{t}$ is a martingale with a mean of zero, and $M_{t}$ is independent of the discount factor $W_{t}$. Using this notation, the discounted surplus can be rewritten as

$$
U_{t}^{(W)}=u+\theta \lambda p_{1} \int_{0}^{t} W_{s} d s+H_{t},
$$

where $H_{t}=\int_{0}^{t} W_{s} d M_{s}$ is also a martingale with a mean of zero.

First, we will prove the convergence of $\int_{0}^{t} W_{s} d s$. Since $W_{s}$ is nonnegative for all $s \geq 0$, the monotone convergence theorem implies

$$
\int_{0}^{t} W_{s} d s \uparrow \int_{0}^{+\infty} W_{s} d s
$$

because

$$
E\left[\int_{0}^{+\infty} W_{s} d s\right]=\frac{1}{\gamma_{1}}
$$


The random variable $\int_{0}^{\infty} W_{s} d s$ is $P-$ a.s. finite, which proves the convergence of $\int_{0}^{t} W_{s} d s$. Next, we will prove the convergence of $H_{t}$. Note that $\left\{H_{t}\right\}$ is an $L_{2}$-bounded martingale; the result follows from a standard result. Therefore, there exists a finite random variable $H_{\infty}$ such that

$$
\lim _{t \rightarrow \infty} H_{t} \stackrel{\text { a.e. }}{=} H_{\infty} \quad \text { and } \quad E H_{\infty}=E H_{0}=0
$$

From the above results, we see that there exists a finite random variable $U_{\infty}^{(W)}$ such that

$$
\lim _{t \rightarrow+\infty} U_{t}^{(W)} \stackrel{\text { a.e. }}{=} U_{\infty}^{(W)} \text { and } E\left(U_{\infty}^{(W)}\right)=\frac{\theta \lambda p_{1}}{\gamma_{1}}+u \text {. }
$$

The characteristic function of $U_{\infty}^{W}$ is obtained by straightforward calculation.

Remark 1: It is well known that for the classical compound Poisson model, if $\theta>0$, the surplus process will move toward $+\infty$ with a probability 1 as the time $t \rightarrow \infty$. This means that in the classical compound Poisson model, if we let the surplus process continue even when the ruin occurs, the surplus process will eventually become very large (tend to infinity with probability 1 as time tends to infinity). See Dassios and Embrechts [4], for further detailed discussions on this issue. Theorem 1 tells us that if we include the interest effect in the model, this is not the case.

\section{AN EXPRESSION OF RUIN PROBABILITY}

Ruin probability has been one of the most important research topics for almost a century, ever since the pioneering work of Lundberg [7,8]. Elegant mathematics has been developed on this topic.

In this section, we will use the result of Theorem 1 to give an expression for the ruin probability. Let $T^{(u)}=\inf \left\{t ; U_{t}^{(W)}<0\right\}$ and $\psi(u)$ denote the ruin probability of model (2.5). Then,

$$
\psi(u)=P\left\{T^{(u)}<\infty \mid U^{(W)}(0)=u\right\} .
$$

Let $U_{t}=(1+\theta) \lambda p_{1} t-\sum_{i=1}^{N(t)} X_{i}$; then,

$$
U_{t}^{(W)}=u+\int_{0}^{t} W_{s} d U_{s}
$$

Let

$$
V_{t}^{(W)}=W_{t}^{-1} \int_{t}^{+\infty} W_{s} d U_{s}
$$


then,

$$
U_{\infty}^{(W)}=U_{t}^{(W)}+W_{t} V_{t}^{(W)} .
$$

Notice that $U_{t}$ has stationary and independent increments and that it is independent of $W_{t}$. If we assume

$$
-\ln \left(W_{t}\right) \text { has stationary and independent increments, }
$$

then we can obtain that

$$
V_{t}^{(W)}=\int_{0}^{+\infty} \widetilde{W}_{s} d \widetilde{U}_{s} \stackrel{d}{=} U_{\infty}^{(W)}-u,
$$

where

$$
\widetilde{W}_{s}=\frac{W_{t+s}}{W_{t}} \stackrel{d}{=} W_{s}, \quad \tilde{U}_{s}=U_{t+s}-U_{t} \stackrel{d}{=} U_{s}
$$

Remark 2: Because we do not require that $W_{t}$ be nonincreasing in this article, any Levy processes will satisfy assumption (*).

Lemma 1: The process $\left\{U_{t}^{(W)}\right\}$, condition on $\sigma\left(W_{s}: 0 \leq s<t\right)$, has an independent increment, and under assumption (*),

$$
\left[\left(U_{t+s}^{(W)}-U_{s}^{(W)}\right) \mid \sigma\left(W_{v}: 0 \leq v<s\right)\right] \stackrel{d}{=}\left(U_{t}^{(W)}-u\right) W_{s},
$$

where $\stackrel{d}{=}$ means that both the left-hand side and the right-hand side of (4.4) have the same distribution.

Proof: For $t>s>0$,

$$
\begin{aligned}
U_{t}^{(W)}-U_{s}^{(W)} & =(1+\theta) \lambda p_{1} \int_{s}^{t} W_{v} d v-\sum_{i=N(s)+1}^{N(t)} X_{i} W_{T_{i}} \\
& =(1+\theta) \lambda p_{1} \int_{s}^{t} W_{s} \frac{W_{v}}{W_{s}} d v-\sum_{i=N(s)+1}^{N(t)} X_{i} W_{s} \frac{W_{T_{i}}}{W_{s}} \\
& =W_{s}\left[\int_{s}^{t} \frac{W_{v}}{W_{s}} d\left((1+\theta) \lambda p_{1} v-\sum_{i=N(s)+1}^{N(v)} X_{i}\right)\right] .
\end{aligned}
$$

Notice that $\left\{W_{t}\right\}$ is independent of $\{N(t): t \geq 0\}$ and $\left\{X_{i}: i=1,2, \ldots\right\}$, so $(1+\theta) \lambda p_{1} v-\sum_{i=N(s)+1}^{N(v)} X_{i}$ has independent increments and is independent of $W_{s}$. Therefore, this lemma holds. 
From the above results, we have the following theorem.

THEOREM 2: Under assumption $(*)$, let $F_{\infty}(x)$ be the distribution function of $U_{\infty}^{(W)}-u$. Then,

$$
\psi(u)=\frac{F_{\infty}(-u)}{E\left(F_{\infty}\left(-W_{T^{(u)}}^{-1} U_{T^{(u)}}^{(W)} \mid T^{(u)}<\infty\right)\right)} .
$$

Proof:

$$
\begin{aligned}
F_{\infty}(-u)= & P\left(U_{\infty}^{(W)}<0\right)=P\left(U_{\infty}^{(W)}<0, T^{(u)}<\infty\right) \\
& =P\left\{U_{T^{(u)}}^{(W)}+W_{T^{(u)}} V_{T^{(u)}}^{(W)}<0 ; T^{(u)}<\infty\right\} \\
& =P\left\{W_{T^{(u)}}^{-1} U_{T^{(u)}}^{(W)}+V_{T^{(u)}}^{(W)}<0, T^{(u)}<\infty\right\} \\
& =P\left\{V_{T^{(u)}}^{(W)}<-W_{T^{(u)}}^{-1} U_{T^{(u)}}^{(W)}, T^{(u)}<\infty\right\} \\
& =\int_{0}^{\infty} P\left\{V_{T^{(u)}}^{(W)}<-W_{T^{(u)}}^{-1} U_{T^{(u)}}^{(W)} \mid T^{(u)}=t\right\} P^{T^{(u)}} d t \\
& =\int_{0}^{\infty} P\left\{U_{\infty}^{(W)}-u<-W_{t}^{-1} U_{t}^{(W)}\right\} P^{T^{(u)}} d t \\
& =\int_{0}^{\infty} F_{\infty}\left(-W_{t}^{-1} U_{t}^{(W)}\right) P^{T^{(u)}} d t=E\left[F_{\infty}\left(-W_{T^{(u)}}^{-1} U_{T^{(u)}}^{(W)}\right) I_{\left\{T^{(u)}<\infty\right\}}\right] \\
= & E\left[F_{\infty}\left(-W_{T^{(u)}}^{-1} U_{T^{(u)}}^{(W)} \mid T^{(u)}<\infty\right] P\left(T^{(u)}<\infty\right)\right. \\
= & E\left[F_{\infty}\left(-W_{T^{(u)}}^{-1} U_{T^{(u)}}^{(W)}\right) \mid T^{(u)}<\infty\right] \psi(u) .
\end{aligned}
$$

Here, we have used the notation $V_{t}^{(W)} \stackrel{d}{=} U_{\infty}^{(W)}-u$. From this, the result is proved.

We say that a distribution function $F(x)$ is a new worse than used (NWU) distribution if $F(x)$ is a distribution function (d.f.) of a nonnegative random variable and $\bar{F}(x)=1-F(x)$ satisfies that $\bar{F}(x) \bar{F}(y) \leq \bar{F}(x+y)$ for $x \geq 0$ and $y \geq 0$. We say that a distribution function $F(x)$ is a new better than used (NBU) distribution if $F(x)$ is a d.f. of a nonnegative random variable and satisfies that $\bar{F}(x) \bar{F}(y) \geq \bar{F}(x+y)$ for $x \geq 0$ and $y \geq 0$.

Proposition 1: Under assumptions (3.1) and (*), we have the following:

1. If $X_{i}$ 's distribution function is $N W U$, then, in this case, we have that

$$
\psi(u) \leq \frac{F_{\infty}(-u)}{E\left(F_{\infty}\left(X_{i}\right)\right)} .
$$


2. If $X_{i}$ 's distribution function is $N B U$, then, in this case, we have

$$
\psi(u) \geq \frac{F_{\infty}(-u)}{E\left(F_{\infty}\left(X_{i}\right)\right)} .
$$

3. If $X_{i}$ 's distribution function is exponential, then, in this case, we have

$$
\psi(u)=\frac{F_{\infty}(-u)}{E\left(F_{\infty}\left(X_{i}\right)\right)} .
$$

Proof: We only give the proof for part 1 because part 2 can be proved by the same method, and part 3 can be obtained from parts 1 and 2 because the exponential distribution is both NWU and NBU.

$$
\begin{aligned}
& P\left\{U_{\infty}^{(W)}-u<-W_{T^{(u)}}^{-1} U_{T^{(u)}}^{(W)} \mid T^{(u)}<\infty\right\} \\
& \quad=P\left\{U_{\infty}^{(W)}-u<-W_{T^{(u)}-}^{-1} U_{T^{(u)}}^{(W)}+X_{i} \mid X_{i}>W_{T^{(u)}-}^{-1} U_{T^{(u)}-}^{(W)}\right\} \\
& \quad=P\left\{X_{i}>U_{\infty}^{(W)}-u+W_{T^{(u)}-}^{-1} U_{T^{(u)}-}^{(W)} \mid X_{i}>W_{T^{(u)}-}^{-1} U_{T^{(u)}-}^{(W)}\right\}
\end{aligned}
$$

where $T^{(u)-}$ means the time just before ruin.

When $U_{\infty}^{(W)}-u>0$, we can get from the distribution of $X_{i}$ is NBU, that the above probability is less than $P\left\{X_{i}>U_{\infty}^{(w)}-u\right\}$-that is, the value $E\left(F_{\infty}\left(X_{i}\right)\right)$. When $U_{\infty}^{(w)}-u<0$, the above probability is 1 and the value $P\left\{X_{i}>U_{\infty}^{(w)}-u\right\}$ also equals one.

After all, when the distribution of $X_{i}$ is NBU, we have

$$
P\left\{U_{\infty}^{(W)}-u<-W_{T^{(u)}}^{-1} U_{T^{(u)}}^{(W)} \mid T^{(u)}<\infty\right\} \leq E\left(F_{\infty}\left(X_{i}\right)\right),
$$

so we can get part 1 .

Remark 3:

1. When $F_{\infty}(0)>0$, we have

$$
F_{\infty}(-u) \leq \psi(u) \leq \frac{F_{\infty}(-u)}{F_{\infty}(0)} .
$$

2. From the expression

$$
F_{\infty}(-u)=P\left\{U_{\infty}^{(W)}-u \leq-u\right\}=P\left\{U_{\infty}^{(W)} \leq 0\right\},
$$

we can say that $F_{\infty}(-u)$ is just the probability of ruin for good (i.e., the probability of the surplus tends to negative infinity).

3. It is interesting that the ruin probability can be determined by the limit distribution of the discounted surplus process. Theorem 2 indicates the relationship between the ruin probability and the limit distribution of the discounted surplus process. 


\section{CONSTANT DISCOUNT RATE AND EXPONENTIAL CLAIM SIZE}

We consider a special case in which the discount rate is a constant $\delta$ and the claim size is an exponentially distributed random variable. This case was treated in Sundt and Teugels [13]. We will obtain this result as a special case of our results in the previous sections. In this section, we will assume that $W_{t}=e^{-\delta t}$ and $X_{i}$ is exponentially distributed with a mean of $p_{1}$. We will first give Lemma 2 .

Lemma 2: The discounted value of all of the claim amounts occurring in the time interval $(0, t], S(t)=\sum_{i=1}^{N(t)} X_{i} e^{-\delta T_{i}}$, converges to a random variable $S(+\infty)$. Furthermore, $S(+\infty)$ has a gamma distribution with parameters $\left(\lambda / \delta, p_{1}^{-1}\right)$.

Proof: According to the properties of the filtered Poisson process, we can derive the characteristic function of $S(t)$ by (see Snyder [12])

$$
E\left(e^{i \tau S(t)}\right)=\exp \left[\lambda \int_{0}^{t} E\left(e^{i \tau X_{i} \exp (-\delta s)}-1\right) d s\right]=\exp \left(\lambda \int_{0}^{t} \frac{i \tau e^{-\delta s}}{p_{1}^{-1}-i \tau e^{-\delta s}} d s\right) .
$$

Let $t \rightarrow+\infty$; then,

$$
\begin{aligned}
\lambda \int_{0}^{+\infty} \frac{i \tau e^{-\delta s}}{p_{1}^{-1}-i \tau e^{-\delta s}} d s & =\frac{\lambda}{\delta} \int_{0}^{\tau} \frac{i d y}{p_{1}^{-1}-i y} \\
& =\frac{\lambda}{\delta}\left[\ln \left(p_{1}^{-1}\right)-\ln \left(p_{1}^{-1}-i \tau\right)\right]=\frac{\lambda}{\delta} \ln \left(\frac{p_{1}^{-1}}{p_{1}^{-1}-i \tau}\right) .
\end{aligned}
$$

The characteristic function of $S(+\infty)$ is then

$$
E\left(e^{i \tau S(\infty)}\right)=\left(\frac{p_{1}^{-1}}{p_{1}^{-1}-i \tau}\right)^{\lambda / \delta} .
$$

This is the characteristic function of a gamma-distributed random variable with parameters $\left(\lambda / \delta, p_{1}^{-1}\right)$. Therefore, the lemma holds.

As explained in Remark 4, part 2 that follows, the following theorem gives the same result as that in Sundt and Teugels [13].

THEOREM 3: Under the assumptions in this section, the ruin probability $\psi(u)$ of model (2.5) has the following expression:

$$
\psi(u)=\frac{1-F_{\Gamma\left(\lambda \delta^{-1}, p_{1}^{-1}\right)}\left(u+\frac{(1+\theta) \lambda}{\delta p_{1}^{-1}}\right)}{1-E\left\{F_{\Gamma\left(\lambda \delta^{-1}, p_{1}^{-1}\right)}\left(\frac{(1+\theta) \lambda}{\delta p_{1}^{-1}}-X_{i}\right)\right\}},
$$

where $F_{\Gamma\left(\lambda \delta^{-1}, p_{1}^{-1}\right)}(\cdot)$ denotes the distribution function of the $\Gamma$ random variable with the parameters $\left(\lambda \delta^{-1}, p_{1}^{-1}\right)$. 
Proof: Under the assumptions in this section, model (2.5) becomes

$$
U_{t}^{(W)}=u+\frac{1+\theta}{\delta} \lambda p_{1}(1-\exp (-\delta t))-\sum_{i=1}^{N(t)} X_{i} e^{-\delta T_{i}}
$$

From Lemma 2, we know that

$$
\sum_{i=1}^{N(t)} X_{i} e^{-\delta T_{i}} \underset{t \rightarrow \infty}{\stackrel{\text { a.e. }}{t \rightarrow \infty}} S(+\infty),
$$

where $S(+\infty)$ is gamma-distributed with the parameters $\left(\lambda / \delta, p_{1}^{-1}\right)$, (i.e., $u+$ $[(1+\theta) / \delta] p_{1} \lambda-U_{t}^{(W)}$ converges to a $\Gamma$ random variable with the parameters $\left.\left(\lambda / \delta, p_{1}^{-1}\right)\right)$. Then, using Theorem 2 , the result is proved.

\section{Remark 4:}

1. Notice that

$$
\begin{aligned}
1-E & \left\{F_{\Gamma}\left(\frac{\lambda}{\delta}, \frac{1}{p_{1}}\right)\left(\frac{1+\theta}{\delta} \lambda p_{1}-X_{i}\right)\right\} \\
= & 1-\int_{0}^{[(1+\theta) / \delta] \lambda p_{1}} \frac{1}{p_{1}} e^{-y / p_{1}} \\
& \times \int_{0}^{[(1+\theta) / \delta] \lambda p_{1}-y} \frac{\left(\frac{1}{p_{1}}\right)^{\lambda / \delta}}{\Gamma\left(\frac{\lambda}{\delta}\right)} e^{-\left(x / p_{1}\right)} x^{(\lambda / \delta)-1} d x d y \\
= & \int_{[(1+\theta) / \delta] \lambda p_{1}}^{\infty} \frac{\left(\frac{1}{p_{1}}\right)^{\lambda / \delta}}{\Gamma\left(\frac{\lambda}{\delta}\right)^{-\left(x / p_{1}\right)} x^{(\lambda / \delta)-1} d x+e^{-[(1+\theta) / \delta] \lambda}} \\
& \times \frac{\delta}{\lambda}\left(\frac{(1+\theta) \lambda p_{1}}{\delta}\right)^{\lambda / \delta} \frac{\left(\frac{1}{p_{1}}\right)^{\lambda / \delta}}{\Gamma\left(\frac{\lambda}{\delta}\right)}
\end{aligned}
$$

Therefore, the result in Theorem 3 is the same as that in Sundt and Teugels [13].

2. The result in Theorem 3 was obtained in Sundt and Teugels [13] by solving the associated renewal equation. We have obtained the result here by using the limit distribution of the surplus process. Therefore, we have, in fact, provided more information than in Sundt and Teugels [13]; that is, we have also proved that the limit distribution of the discounted surplus process is a gamma distribution. 


\section{UPPER BOUNDS FOR RUIN PROBABILITY}

In this section, we will derive the upper bounds for ruin probability. The main results are given in the following two theorems. The techniques used in the proof are similar to those in Yang [14].

THEOREM 4: Suppose that $G(\cdot)$ is a nonnegative measurable function, $U_{t}^{(W)}$ is the discounted surplus of the insurance company at time $t$, and $G\left(U_{t}^{(W)}\right)$ is a supermartingale with respect to $\sigma$-field $\mathcal{F}_{t} \supset \sigma\left(U_{s}^{(W)} \mid 0 \leq s<t\right)$. Then,

$$
\psi(u) \leq \frac{G(u)}{E\left(G\left(U_{T^{(u)}}^{(W)}\right) \mid T^{(u)}<\infty\right)} .
$$

If $\lim _{t \rightarrow \infty} E\left(G\left(U_{t}^{(W)}\right) \mid T^{(u)}>t\right)=0$ and $G\left(U_{t}^{(W)}\right)$ is an $\mathcal{F}_{t}$ martingale, then

$$
\psi(u)=\frac{G(u)}{E\left(G\left(U_{T^{(u)}}^{(W)}\right) \mid T^{(u)}<\infty\right)},
$$

where $T^{(u)}$ is the same as earlier.

Proof: $T^{(u)}$ is an $\mathcal{F}_{t}$ stopping time. For $\forall t>0, T^{(u)} \wedge t$ is a bound $\mathcal{F}_{t}$ stopping time. Using Doob's bounded stopping time theorem, we have

$$
\begin{aligned}
G(u) & =E\left(G\left(U_{0}^{(W)}\right)\right) \geq E\left(G\left(U_{T^{(u)}(W t}^{(W)}\right)\right) \\
& =E\left(G\left(U_{T^{(u)}}^{(W)}\right) \mid T^{(u)}<t\right) \cdot P\left\{T^{(u)}<t\right\}+E\left(G\left(U_{T^{(u)}}^{(W)}\right) \mid T^{(u)} \geq t\right) \cdot P\left\{T^{(u)} \geq t\right\} .
\end{aligned}
$$

Let $t \rightarrow+\infty$; by the positive of $G(\cdot)$, we have

$$
G(u) \geq E\left(G\left(U_{T^{(u)}}^{(W)}\right) \mid T^{(u)}<\infty\right) \psi(u) .
$$

Therefore, (6.1) holds.

Moreover, if $\lim _{t \rightarrow+\infty} E\left(G\left(U_{T^{(u)}}^{(W)}\right) \mid T^{(u)}>t\right)=0$ and $G\left(U_{t}^{(W)}\right)$ is an $\mathcal{F}_{t}$ martingale, then (6.2) also holds.

THeOREm 5: If, for all $t \geq 0,0<W_{t}<1$ a.e., assume that there exists $R>0$ such that

$$
E\left(e^{R X_{i}}-1\right)=R(1+\theta) E\left(X_{i}\right)=R(1+\theta) p_{1} .
$$

Then,

$$
\psi(u) \leq \frac{\exp (-R u)}{E\left(\exp \left(-R U_{T^{(u)}}^{(W)}\right) \mid T^{(u)}<\infty\right)} \leq e^{-R u} .
$$

Proof: For all $x \in(0,1], y>0$,

$$
\sum_{n=1}^{\infty} \frac{(x y)^{n}}{n !} \leq \sum_{n=1}^{\infty} \frac{x(y)^{n}}{n !}
$$


holds; that is,

$$
e^{x y}-1 \leq x\left(e^{y}-1\right) .
$$

Let $h(r)=E\left(e^{r X_{i}}-1\right)$. Because for all $t>0,0<W_{t}<1$ a.e, we then have

$$
h\left(R W_{s}\right) \leq W_{s} h(R) .
$$

Let

$$
N_{t}=\exp \left\{R \sum_{i=1}^{N(t)} X_{i} W_{T_{i}}-R(1+\theta) p_{1} \lambda \int_{0}^{t} W_{s} d s-R u\right\}
$$

and

$$
\mathcal{F}_{t}^{\prime}=\sigma\left(N_{s}: s \leq t\right) \vee \sigma\left(W_{\tau}: \tau>0\right) .
$$

Using a similar argument as that in Deng and Liang [6, pp. 329-332] (also see Snyder [12]) and after some calculations, we obtain

$$
E\left[\exp \left\{R \sum_{i=N(s)+1}^{N(t)} X_{i} W_{T_{i}}\right\} \mid \sigma\left(W_{\tau}: \tau \geq 0\right)\right]=\exp \left(\lambda \int_{s}^{t} h\left(R W_{\tau}\right) d \tau\right),
$$

so

$$
\begin{aligned}
E\left(N_{t} \mid \mathcal{F}_{s}^{\prime}\right) & =N_{s} E\left[\exp \left(R \sum_{i=N(s)+1}^{N(t)} X_{i} W_{T_{i}}-R(1+\theta) p_{1} \lambda \int_{s}^{t} W_{\tau} d \tau\right) \mid \mathcal{F}_{s}^{\prime}\right] \\
& =N_{s} \exp \left(\lambda \int_{s}^{t} h\left(R W_{\tau}\right) d \tau-R(1+\theta) p_{1} \lambda \int_{s}^{t} W_{\tau} d \tau\right) \\
& \leq N_{s} \exp \left(\lambda \int_{s}^{t} W_{\tau} h(R) d \tau-R(1+\theta) p_{1} \lambda \int_{s}^{t} W_{\tau} d \tau\right) \\
& =N_{s} .
\end{aligned}
$$

Therefore, $N_{t}$ is an $\mathcal{F}_{t}^{\prime}$ supermartingale. From Theorem 4, we have

$$
\psi(u) \leq \frac{\exp (-R u)}{E\left(\exp \left(-R U_{T^{(u)}}^{(W)}\right) \mid T^{(u)}<\infty\right)},
$$

where $R>0$ and $U_{T^{(u)}}^{(W)}<0$. Note that $E\left[\exp \left(-R U_{T^{(u)}}^{(W)}\right) \mid T^{(u)}<\infty\right]>1$. Therefore, the result of the theorem holds.

\section{UPPER BOUNDS FOR RUIN PROBABILITY IN THE CASE OF NEGATIVE LOADING}

It is well known that, in the classical model, if the loading is negative, the ruin becomes certain. We will show below that this is not the case in the model with stochastic interest rate. We first introduce the following sets: 


$$
\begin{aligned}
D & =\left\{r>0: E\left[\exp \left\{r X_{1}\right\}\right]<+\infty\right\}, \\
D_{t} & =\left\{r>0: \int_{0}^{t}\left[E\left\{\exp \left(r W_{s} X_{1}\right)\right\}-1\right] d s<+\infty\right\} \text { for } 0<t \leq+\infty .
\end{aligned}
$$

From the definition, we can easily see that $D$ is an interval of the form $(0, \bar{r})$ or $(0, \bar{r}]$. It is not difficult to see that for $0<t<\infty$, we have $D_{t}=D$ if $D=(0, \bar{r}]$, and if $D=(0, \bar{r})$ with $\bar{r}<\infty$, then $D_{t}=(0, \bar{r})$ or $(0, \bar{r}]$. Furthermore, if $0<t$ and $u<\infty$, then $D_{t}=D_{u}$.

It is not difficult to prove that the assumption $E\left[\int_{0}^{+\infty} W_{s} d s\right]=1 / \gamma_{1}$ implies that $D_{\infty}=D_{t}$.

The following theorem provides us with an upper bound for ruin probability in the case of negative safety loading.

Theorem 6: Assume that the safety loading $\theta \leq 0$ and $0<t<\infty$. Then, we have the following results:

$$
\begin{aligned}
& P(T \leq t) \leq \inf _{r \geq 0} \exp \left\{\lambda \int_{0}^{t} E\left[\exp \left\{r W_{s} X_{1}\right\}-1-(1+\theta) r W_{s} X_{1}\right] d s-r u\right\}, \\
& P(T<\infty) \leq \inf _{r \geq 0} \exp \left\{\lambda \int_{0}^{\infty} E\left[\exp \left\{r W_{s} X_{1}\right\}-1-(1+\theta) r W_{s} X_{1}\right] d s-r u\right\} .
\end{aligned}
$$

Proof: For $r \in D_{t}$, let $h(r)=E\left[\exp \left(r X_{1}\right)\right]-1$ and define

$$
\begin{aligned}
M_{t} & =\exp \left\{r \sum_{i=1}^{N(t)} W_{T_{i}} X_{i}-\lambda \int_{0}^{t} h\left(r W_{s}\right) d s\right\} \\
& =\exp \left\{r \sum_{i=1}^{N(t)} W_{T_{i}} X_{i}+\lambda t-\lambda \int_{0}^{t} E\left[\exp \left\{r W_{s} X_{1}\right\}\right] d s\right\} .
\end{aligned}
$$

With the same arguments as in the proof of Theorem 5, it is not difficult to check that $M_{t}$ is a martingale with respect to $\mathcal{F}_{t}$, where

$$
\mathcal{F}_{t}=\sigma\left\{M_{s}: s \leq t\right\} \vee \sigma\left\{W_{\tau}: \tau \geq 0\right\}
$$

Applying Doob's bounded stopping time theorem to the martingale $\left\{M_{t}: t \in R_{+}\right\}$for the stopping time $T \wedge t, 0<t<\infty$, we have

$$
\begin{aligned}
1 & =E\left[M_{0}\right]=E\left[M_{T \wedge t}\right] \geq E\left[M_{T} I_{\{T \leq t\}}\right] \\
& =E\left[\exp \left\{r \sum_{i=1}^{N(T)} W_{T_{i}} X_{i}+\lambda T-\lambda \int_{0}^{T} E\left[\exp \left\{r W_{s} X_{1}\right\}\right] d s\right] I_{\{T \leq t\}}\right] \\
& \geq E\left[\exp \left\{r u-\lambda \int_{0}^{T} E\left[\exp \left\{r W_{s} X_{1}\right\}-1-(1+\theta) r W_{s} X_{1}\right] d s\right\} I_{\{T \leq t\}}\right] \\
& \geq \exp \left\{r u-\sup _{0 \leq s \leq t} \lambda \int_{0}^{s} E\left[\exp \left\{r W_{v} X_{1}\right\}-1-(1+\theta) r W_{v} X_{1}\right] d v\right\} P(T \leq t),
\end{aligned}
$$


where $I_{\{A\}}$ denotes the indicator function of set $A$, and we have used the fact that, at the ruin time $T, \sum_{i=1}^{N(t)} W_{T_{i}} X_{i}>u+(1+\theta) \lambda p_{1} \int_{0}^{T} W_{s} d s$.

Hence, if $r \geq 0$,

$$
P(T \leq t) \leq \sup _{0 \leq s \leq t} \exp \left\{\lambda \int_{0}^{s} E\left[\exp \left\{r W_{v} X_{1}\right\}-1-(1+\theta) r W_{v} X_{1}\right] d v-r u\right\} .
$$

Therefore,

$$
P(T \leq t) \leq \inf _{r \geq 0} \sup _{0 \leq s \leq t} \exp \left\{\lambda \int_{0}^{s} E\left[\exp \left\{r W_{v} X_{1}\right\}-1-(1+\theta) r W_{v} X_{1}\right] d v-r u\right\}
$$

From the inequality $e^{x}-1 \geq x, \theta \leq 0$, and that $W_{s}$ and $X_{1}$ are positive random variables, we have that

$$
E\left[\exp \left\{r W_{s} X_{1}\right\}-1-(1+\theta) r W_{s} X_{1}\right] \geq 0 .
$$

Hence, (7.6) can be rewritten as

$$
P(T \leq t) \leq \inf _{r \geq 0} \exp \left\{\lambda \int_{0}^{t} E\left[\exp \left\{r W_{s} X_{1}\right\}-1-(1+\theta) r W_{s} X_{1}\right] d s-r u\right\} .
$$

From this, the theorem follows.

Notice that the upper bounds in theorem 6 only make sense if the right-hand sides of (7.6) and (7.7) are smaller than one. We will address this problem now.

For $0<t \leq \infty$, let

$$
\begin{aligned}
g_{t}(r) & =\lambda \int_{0}^{t} E\left[\exp \left\{r W_{s} X_{1}\right\}-1-(1+\theta) r W_{s} X_{1}\right] d s-r u, \\
\Phi_{t}(r) & =\exp \left\{g_{t}(r)\right\} .
\end{aligned}
$$

Proposition 2: Suppose that $0<t \leq \infty$ and the safety loading $\theta \leq 0 . \Phi_{t}(r)$ is then a convex mapping on $R_{+}$and is finite on $D_{t}$.

Proof: Similar to Lemma 4.5 of Delbaen and Haezendonck [5], we can prove the following:

If

$$
\frac{d^{-}}{d r} \Phi_{t}(r) \neq \frac{d^{+}}{d r} \Phi_{t}(r)
$$


then

$$
\frac{d^{-}}{d r} \Phi_{t}(r)<\frac{d^{+}}{d r} \Phi_{t}(r)
$$

If

$$
\frac{d^{-}}{d r} \Phi_{t}(r)=\frac{d^{+}}{d r} \Phi_{t}(r),
$$

then

$$
\frac{d^{2}}{d r^{2}} \Phi_{t}(r)>0
$$

From this, it follows that $\Phi_{t}(r)$ is a convex mapping on $R_{+}$. It is obvious that $\Phi_{t}(r)$ is finite on $D_{t}$.

The above analysis indicates that $\inf _{r \geq 0} \Phi_{t}(r)<1$ if the right derivative of $\Phi_{t}(r)$ at $r=0$ is strictly negative. However,

$$
\begin{aligned}
\frac{d^{+}}{d r} \Phi_{t}(0) & =\left.\left(\lambda \int_{0}^{t} E\left[W_{s} X_{1}\left[\exp \left(r W_{s} X_{1}\right)-(1+\theta)\right]\right] d s-u\right) \exp \left\{g_{t}(r)\right\}\right|_{r=0} \\
& =-\lambda \theta \int_{0}^{t} E\left(W_{s} X_{1}\right) d s-u=-\lambda \theta p_{1} \int_{0}^{t} E\left(W_{s}\right) d s-u
\end{aligned}
$$

where the last equality holds because $X_{1}$ and $W_{s}$ are independent. This leads to the following proposition.

Proposition 3: If $0<t<\infty$ and

$$
0 \geq \theta>-\frac{u}{\lambda p_{1} \int_{0}^{t} E\left(W_{s}\right) d s},
$$

there exists a real number $r_{t}>0$ such that

$$
P(T<t) \leq \Phi_{t}\left(r_{t}\right)=\inf _{r \geq 0} \Phi_{t}(r)<1 .
$$

Furthermore, if

$$
0 \geq \theta>-\frac{u}{\lambda p_{1} \int_{0}^{\infty} E\left(W_{s}\right) d s},
$$

there exists a real number $r_{\infty}>0$, such that

$$
P(T<\infty) \leq \Phi_{\infty}\left(r_{\infty}\right)=\inf _{r \geq 0} \Phi_{\infty}(r)<1 .
$$


We conclude this section by giving two special cases. First, we consider the classical compound Poisson model $\left(W_{s}=1\right)$. In this case, if the risk loading $\theta$ is negative, the ultimate ruin probability will be one. The upper bound for the ruin probability at a finite time, say before time $t$, is nontrivial if

$$
t<-\frac{u}{\lambda p_{1} \theta} \text { and } \theta \leq 0 .
$$

The second case is the situation with a constant interest force. In this case, $W_{s}=e^{-\delta s}$. A detailed discussion on this case can be found in Boogaert and Crijins [3].

\section{Acknowledgments}

The authors would like to thank Professor Elias Shiu for pointing out an error and some problems with the model in the earlier version of this article. This work was supported by grants from Research Grants Council of HKSAR (project no. HKU 7139/01H).

\section{References}

1. Aczel, J. \& Dhombres, J. (1989). Functional equations in several variables: With applications to mathematics, information theory and to the natural and social sciences. New York: Cambridge University Press.

2. Asmussen, S. (2000). Ruin probabilities. Singapore: World Scientific.

3. Boogaert, P. \& Crijins, V. (1987). Upper bounds on ruin probabilities in case of negative loadings and positive interest rate. Insurance: Mathematics and Economics 6: 221-232.

4. Dassios, A. \& Embrechts, P. (1989). Martingales and insurance risk. Communications in StatisticsStochastic Models 5: 181-217.

5. Delbaen, F. \& Haezendonck, J. (1987). Classical risk theory in an economic environment. Insurance: Mathematics and Economics 6: 85-116.

6. Deng, Y.L. \& Liang, Z.S. (1992). Stochastic point processes and applications. Beijing: Science Publisher (in Chinese).

7. Lundberg, F. (1903). I. Approximerad Framstallning af Sannolikhetsfunktionen. II. Aterforsakring af Kollektivrisker. Uppsala: Almqvist and Wiksells.

8. Lundberg, F. (1926). Forsakringsteknisk Riskutjamning. Stockholm: F. Englunds Boktryckeri.

9. Norberg, R. (1999). Ruin problems with asset and liabilities of diffusion type. Stochastic Processes and Their Applications 81: 255-269.

10. Paulsen, J. (1998). Ruin theory with compounding assets-A survey. Insurance: Mathematics and Economics 22: 3-16.

11. Paulsen, J. \& Gjessing, H.K. (1997). Ruin theory with stochastic return on investments. Advances in Applied Probability 29: 965-985.

12. Snyder, D.L. (1975). Random point processes. New York: Wiley.

13. Sundt, B. \& Teugels, J.L. (1995). Ruin estimates under interest force. Insurance: Mathematics and Economics 16: 7-22.

14. Yang, H. (1999). Non-exponential bounds for ruin probability with interest effect included. Scandinavian Actuarial Journal 1999: 66-79.

15. Yang, H. \& Zhang, L. (2001). The joint distribution of surplus immediately before ruin and the deficit at ruin under interest force. North American Actuarial Journal 5(3): 92-103. 